\title{
What the Seller Won't Tell You: Persuasion and Disclosure in Markets
}

Paul Milgrom ${ }^{1}$

Stanford University

February, 2007

Imagine that you are considering an investment in a new public offering of a firm’s shares. The firm’s officers have visited your city to make a presentation which included an audited financial statement, an earnings forecast was reviewed by its prestigious investment bankers, and an impressive demonstration of it new technology. Should such a presentation convince you to invest? How concerned should you be if no mention was made about a rumored competitor with a different technology? An economist reflecting on these same questions from the standpoint of policy might wonder: Does the firm’s need to convince naturally skeptical investors provide sufficient incentives for it to disclose accurate information in the right form and amounts? Could regulations mandating disclosure sometimes improve the quality of investment decisions? What kinds of regulations are likely to be most helpful, and when?

It is not only in the investment sphere that buyers rely on sellers to supply information. For example, suppose that you are buying a new furnace to replace an old one that is not working well. The salesman displays a chart showing that the projected total life-cycle cost of one particular furnace, including capital costs and fuel usage over the lifetime of the furnace, is lower than for competing models you have considered. Should you worry about what the salesman is not telling you? Might it be that fuel usage costs are less important for an especially well insulated home like yours? What other

\footnotetext{
${ }^{1}$ Special thanks to Will Nelson and Manuj Garg for reading and commenting on a draft of this paper. Financial support has been provided by the National Science Foundation, Award 0648293.
} 
issues didn't the salesman mention that could be relevant to your decision? If you talk to several sellers before buying, can you count on competition among them to bring out all the information you need to make a good decision? How does competition compare to regulation as a mechanism for encouraging sellers to provide valuable information to buyers?

Modern economics textbooks emphasize that the problem of adverse selection can alter the operation of markets in fundamental ways, but little attention has yet been paid to seller's efforts to mitigate that problem. Certainly, sellers of good quality products have a powerful incentive to eliminate adverse selection in order to increase sales. In modern capitalist economies, sellers routinely supply helpful information about their products, sometimes including test results and technical reports. Seller reputations play a role in encouraging honest reporting. In addition, private sector institutions including accounting firms, investment bankers, testing laboratories, hobbyist magazines, and others whose revenue-streams depend on their reputations may provide additional information or evaluate the accuracy of sellers' claims. Public sector institutions also have a role: liability rules and laws against fraud help to ensure that reported information is accurate.

Our purpose in this paper is to study how effectively these institutions mitigate adverse selection. How well does a system of private reporting work? When should we expect all the relevant information to be reported? If testing and reporting are costly, will too little testing and reporting be done? Or too much? When some information is withheld, what sort of information is that? How do rational buyers respond to such withholding? How are prices and welfare affected? What role is there for laws and 
regulations to improve the functioning of markets? We answer some of these questions by studying the theory of persuasion games — games in which one or more players provides verifiable information to others in order to influence the actions they take. ${ }^{2}$

Two themes recur throughout our analyses. First, sophisticated buyers (those who play equilibrium strategies of the persuasion game) are consistently skeptical. When evidence is missing, they view that suspiciously, considering that any missing evidence is likely to be unfavorable to the seller and reduce their purchases accordingly. ${ }^{3}$ The magnitude of this effect depends on what buyers believe about the seller's ability to obtain and communicate the missing information. Second, the combination of consumer skepticism and the seller's ability to reveal information selectively provides a powerful incentive for sellers of all but the worst products to acquire and report information. In the simplest models, this incentive completely solves the adverse selection problem. In equilibrium, sellers voluntarily reveal enough information that sophisticated buyers are able to make the same decisions as if they were fully informed. While empirical evidence about reporting policies is meager, there is one study showing that more frequent issuers of securities engage in more disclosure, which is consistent with the idea that sellers have an extra incentive to supply information. ${ }^{4}$

Does the seller have sufficient incentive to verify its product quality when testing is costly? Jovanovic (1982) finds that, at least sometimes, the answer is surprising: the

\footnotetext{
${ }^{2}$ The term "persuasion games” was coined by Milgrom and Roberts (1986). These games, with their verifiable information, are distinguished from another important class of games-the cheap talk games of Crawford and Sobel (1982) and Farrell and Rabin (1996)) — in which all reported information is unverifiable.

${ }^{3}$ Grossman (1981) and Milgrom (1981) give early general statements of this idea. A still earlier particular application of the idea to securities markets and takeover bidding was developed by Grossman and Hart (1980). Milgrom and Roberts (1986) give the most general statement of the result.

${ }^{4}$ Frankel, McNichols, and Wilson (1995).
} 
seller's incentive to test its product is greater than in any efficient arrangement. To understand this conclusion, fix the buyer's beliefs and strategy for the buyer and consider the seller's decision about whether to conduct $n$ or $n+1$ tests. If the buyer does not know the number of tests run, then the seller can report selectively. If the seller conducts the $n+1^{\text {st }}$ test and its result is positive, the seller can report that favorable result and charge a higher price. If the result is negative, the seller can withhold that. Since the buyer cannot distinguish that from the situation in which the seller had conducted just $n$ tests, the seller can charge the same price in that case as if it had not performed the extra test. So, regardless of the buyer's beliefs, additional testing combined with selective reporting raises the average price, causing a positive transfer on average from the buyer to the seller. That encourages excessive testing. In equilibrium, the sophisticated buyer understands the seller's testing incentives and makes the correct assessments on average, but this negative pecuniary externality generally precludes an equilibrium with an efficient amount of testing.

The problem of selective reporting and its damaging welfare consequences is familiar from news reports. Some of the most striking examples are drawn from the pharmaceutical industry. In one case, Merck’s arthritis drug, Vioxx, was found to double the risk of heart attacks for its users, but although this dangerous side effect was suspected by scientists for years before the drug was banned, there were no full studies confirming that danger and no reports alerting users to the risks. The Vioxx case, particularly the fact that the risks became apparent only after the drug had received FDA approval, has motivated recent policy proposals to change testing and reporting 
requirements for approved drugs. ${ }^{5}$ The Vioxx case is hardly unique. In another recent case, Eli Lilly instructed its salespeople to downplay the risks associated with its schizophrenia drug, Zyprexa, although the effects were severe. ${ }^{6}$

According to some simple theoretical models, if government could impose a requirement of mandatory disclosure preventing the seller from withholding negative test results, then the testing externality would be eliminated and the seller would be led to do an optimal amount of testing. That conclusion, however, does not survive closer scrutiny.

To evaluate policy interventions, it is helpful to have a theory that is consistent with these pharmaceutical examples, and indeed such a theory has been developed. In the theory, the seller may have no verifiable test results to report, but may nevertheless be aware of indications of trouble. The seller decides whether to run certain tests. In the easiest version of the theory, the seller knows in advance what the outcome of any verifiable test will be. Then, there is an equilibrium in which sellers test and reveal "good news" and withhold "bad news" simply by not testing. ${ }^{7}$ For such a strategy to mislead sophisticated buyers, they must be uncertain about which tests can or should be performed. When detailed information is missing, buyers then need to weigh two or more possibilities: either the seller is withholding bad news, or the seller is uninformed, or perhaps testing and reporting are too costly to be worthwhile. With these effects confounding the reporting decision, missing information causes sophisticated buyers to

\footnotetext{
${ }^{5}$ Among the many news stories citing the Vioxx case is one in the New York Times on January 31, 2007, by Gardiner Harris, "FDA Installs Drug Reviews at 18 Months.”

${ }^{6}$ According to the New York Times, Dec 17, 2006, Lilly "kept important information from doctors about Zyprexa's links to obesity and its tendency to raise blood sugar - both known risk factors for diabetes.”

${ }^{7}$ Milgrom (1981). See also Dye (1985) and Verrecchia (1983).
} 
reduce their purchases, but not as much as it would if they were to learn bad news about the product. So, it pays the seller to refrain from conducting and reporting verifiable tests.

These same ideas have application that extend far beyond the pharmaceutical industry. As a second example, suppose that a firm planning a public offering has disgruntled employees who could file an employment discrimination lawsuit. It hardly pays for such a firm to track down the details of that threat, because any news it turns up would most likely discourage investors and reduce its share price. Without a proper legal or institutional framework, market forces alone do not solve the reporting problem.

Legal rules may help. For example, the problem just described would be mitigated by a rule that holds the firm accountable for any unreported information if it should have known that information. In terms of a formal model, "should have known" means that the firm could have run that test at a reasonable cost. In the model, a well-informed firm would have run the test but for its private information that led it to believe that the test result would be unfavorable. Even if the private information is never observed by others, it is a fair inference after the fact that when a firm decides not to conduct an important, inexpensive test and the outcome proves to be unfavorable, that points to the firm having had negative information to underpin its decision. In practice, the "should have known" standard might be replaced by an obligation for management to investigate certain categories of threats, where the ratio of threat cost to evaluation cost is especially high.

Private sector institutions can sometimes address the same sort of issue without the need for potentially politicized regulations. For example, an investment banker may be responsible for doing a thorough investigation of threats to the share value in anticipation of a public offering. For this to be effective, however, the investment banker 
would need ex ante access to the same sorts of information that the court needs only ex post. For example, an investment banker may fail to observe that there is a cheap test to evaluate each of several threats, while the court only needs to pinpoint the threat for which a negative result occurred, which can make ex post verification cheaper and more effective.

High quality sellers benefit when they can credibly make denials even when there is no possibility of verifiable information. For example, a firm issuing shares that has no major pending lawsuits against it benefits if it can claim that without being grouped with others firms who merely claim that there is no verifiable information about pending lawsuits. Accurate reporting can be helped by laws or regulations that mandate disclosures of material negative events that management either knows about, or should have known about. More empirical evidence about the role of laws and regulations mandating disclosure is needed to supplement the general empirical observation that some disclosure regulations do benefit stock markets. ${ }^{8}$

Another interesting variation on persuasion games arises in cases like the furnacebuying example, in which the buyer is so poorly informed about a product that she is not sure which factors are most relevant to her decision. In a monopoly situation, such a buyer is at the mercy of unscrupulous sellers, but the situation is different when there are competing providers of information. ${ }^{9}$ The furnace buyer, for example, may be unaware that it is important to check how well the new furnace will work well with complementary products, such as an existing system of hot air ducts or an existing chimney or cooling system. Information of this kind can often be elicited if the buyer

\footnotetext{
${ }^{8}$ La Porta, Lopez-de-Silanes, and Shleifer (2006).

${ }^{9}$ See Milgrom and Roberts (1986).
} 
inspires competition among sellers, because each will be motivated to explain why its furnace will work better than the competitor's in the buyer's home. Competition in providing information, however, also has its limits. Self-interested furnace sellers would all omit telling the buyer if her problem can be solved most cheaply and effectively simply by adding insulation below the roof rather than by buying any new furnace.

The remainder of this paper reviews these main theoretical points in more detail.

\section{Skepticism and Unraveling}

The simplest persuasion game has two players: an informed seller and a riskneutral uninformed buyer. The seller has private information about a variable $\theta$, which we usually interpret as the one-dimensional quality of its product. We assume that there are $N$ possible qualities $\theta_{1}<\ldots<\theta_{N}$ listed in a set $\Theta=\left\{\theta_{1}, \ldots, \theta_{N}\right\}$. The seller's only move in the game is to make a report $S$ that the uninformed buyer considers in making its purchase decision. Although we will allow the report to be quite abstract, for most of our applications, it is sufficient to think of the report as naming a subset of $\Theta$ and thus directly restricting the possible qualities of the good.

The buyer's decision, $q$, is a scalar that we will usually interpret as the quantity the buyer purchases. Before hearing the seller's report, the buyer believes that the probability of any particular quality level $\theta_{i}$ is $\bar{\pi}_{i}>0$.

Fixing the product price $p$, if the buyer purchases quantity $q$, her payoff is $v(q, \theta)-p q$ and the seller's payoff is $(p-\gamma) q$ for some unit cost $\gamma \in(0, p)$. Let $q_{i}$ 
denote the quantity that maximizes the buyer's payoff in state $\theta_{i}$. (If there are multiple such quantities, take $q_{i}$ to be the largest one.)

Often, we think of quality as raising the marginal value of consumption, but that need not be the case. For example, if higher quality light bulbs use less power and last longer, then the marginal value associated with bulbs bought to replace less power efficient bulbs rises as quality improves, but the marginal value of bulbs bought for inventory, to be used as replacements as bulbs burn out, declines. Over the long term, the higher quality, longer lasting bulbs likely lead to reduced purchases at any given price. This discussion illustrates that the relationship between quality and demand need not be a simple one.

To avoid such complexities, our basic assumption throughout most of this paper is the following:

Assumption A1. Quality increases marginal values: $v_{q \theta} \equiv \partial^{2} v / \partial \theta \partial q>0$.

Unfortunately, this assumption can obfuscate the discussion somewhat and is actually a bit more than is needed for the results of this section so, for now, we replace $A 1$ with two of its useful implications, which we treat as assumptions in their own right. The first is that the optimal quantities are ordered in the same way as the quality index: ${ }^{10}$

Assumption A1(a): $q_{1} \leq \ldots \leq q_{N}$.

\footnotetext{
${ }^{10}$ For a derivation, see Topkis (1978).
} 
The second requires some notation to state precisely. Given beliefs $\pi$ about the quality, the buyer's optimal quantity is $q(\pi)$, which solves $\max _{q} \sum_{j} \pi_{j} v\left(q, \theta_{j}\right)-p q .{ }^{11}$ Then, another implication of A1 is that uncertainty about the quality leads the buyer to compromise, picking some quantity between the optimal amounts called for at the extreme qualities:

Assumption A1(b): Suppose that there exist $\theta_{j}$ and $\theta_{k}$ such that $\pi\left(\theta_{k}\right), \pi\left(\theta_{j}\right)>0$ and $q_{k} \neq q_{j}$. Then, $\min \left\{q_{i} \mid \pi\left(\theta_{i}\right)>0\right\}<q(\pi)<\max \left\{q_{i} \mid \pi\left(\theta_{i}\right)>0\right\}$.

The “compromise” assumption is reasonable for many applications and can be derived from the $v_{q \theta}>0$ condition, but there are plausible situations in which it could fail. For example, suppose that $\theta$ presents information about about driving conditions and $q$ is the type of motor oil used, arranged from least expensive to most expensive. Let $q_{1}$ and $q_{2}$ be different grades of oil that are best for different driving conditions, designated by the two states $\Theta=\left\{\theta_{1}, \theta_{2}\right\}$. Only the seller knows the actual driving conditions and consequently which choice of $q$ is most suitable for the buyer. Quality $q_{3}$ corresponds to a synthetic motor oil that is more profitable for the seller and is effective for all driving conditions. If the buyer is left uninformed about driving conditions, she might find it optimal to buy the most expensive grade, $q_{3}$, which is outside the range $\left\{q_{1}, q_{2}\right\}$. If silence by the seller is feasible, then there is be an equilibrium of the persuasion game in which the seller is always silent and the buyer always purchases $q_{3}$.

\footnotetext{
${ }^{11}$ If there are multiple solutions, then for specificity, we may take $q(\pi)$ to be the largest one.
} 
Assumption A1(b) rules out this example, because it makes it impossible that silence will lead to a choice $q_{3}>\max \left\{q_{1}, q_{2}\right\}$.

So far, we have focused on describing the buyer's full-information preferences. By assumption, however, the buyer is not fully informed about the state and instead relies on the seller for information. When the state is $\theta$, the seller makes some verifiable statement $S$ about $\theta$. The report must be must be feasible given the actual quality, which we take to mean that $S$ must be chosen from $\Omega(\theta)$; the set of feasible reports in each state as determined by $\Omega$ is a parameter of the model. In the example where the seller reports a subset of $S \subset \Theta$, the restriction can be written as $\theta \in S .{ }^{12}$ The key assumption of this section is that it is always possible for a seller to make some verifiable report that distinguishes its quality from lower qualities.

Assumption A2. When the actual state is $\theta$, the seller has available a report $S$ that rules out lower quality types. In symbols: $(\forall \theta)(\exists S \in \Omega(\theta))\left(\forall \theta^{\prime}<\theta\right)\left[S \notin \Omega\left(\theta^{\prime}\right)\right]$.

This assumption would be satisfied, for example, if the seller could always prove the precise quality of its product or if it can prove a tight lower bound on the quality of its product. $^{13}$

The combination of assumptions A1(a\&b) and A2 enables a thorough-going skepticism on the part of the buyer. If the seller chooses not to reveal the detailed state and the buyer knows that it could do so, then the buyer can react by extremely cautious in

\footnotetext{
${ }^{12}$ Formally, $\Omega(\theta) \equiv\left\{S \subseteq 2^{\Theta} \mid \theta \in S\right\}$.

${ }^{13}$ Glazer and Rubinstein (2006) characterize equilibrium in a persuasion game where the buyer's decision is binary and assumption A2 is dropped. There is, as yet, no extension of that model that endogenizes prices or evaluates disclosure policies, so we omit that model from this review.
} 
deciding what to purchase, buying only the minimum proven quantity. We will show below that this is indeed what must happen in equilibrium..

To state that conclusion formally, let $S^{*}(\bullet)$ be the seller's reporting strategy in some equilibrium, so $S^{*}(\theta)$ is what the seller reports when the true state is $\theta$. Similarly, let $q^{*}(\cdot)$ be the buyer's purchasing strategy, so $q^{*}(S)$ is quantity that the buyer purchases when the seller reports $S$. Finally, let $\pi_{S}^{*}$ be the buyer's probability distribution over $\Theta$ after hearing the report $S$.

Definition. A perfect Bayesian equilibrium (hereafter, simply an equilibrium) is a triple $\left(S^{*}, q^{*}, \pi^{*}\right)$ satisfying three conditions:

1. The seller always reports to maximize its net profits. In symbols: for all $\theta \in \Theta$, $S^{*}(\theta) \in \arg \max _{S \in \Omega(\theta)}(p-\gamma) q^{*}(S)$.

2. The buyer maximizes its payoffs, given its beliefs. In symbols, $q^{*}(S)=q\left(\pi_{S}^{*}\right)$.

3. The buyer's beliefs are consistent with the restrictions implied by the sellers' verifiable reports and with Bayes' theorem as applied to the seller’s reporting strategy. In symbols, this implies two restrictions: ${ }^{14}$

a. $\pi_{S}^{*}(\theta)=0$ for $S \notin \Omega(\theta)$

b. For all $S$ in the range of $S^{*}$,

$$
\pi_{S}^{*}(\hat{\theta})=\left\{\begin{array}{lr}
0 & \text { if } S^{*}(\hat{\theta}) \neq S \\
\bar{\pi}(\hat{\theta}) / \sum_{\left\{\theta \mid S^{*}(\theta)=S\right\}} \bar{\pi}(\theta) & \text { otherwise }
\end{array}\right.
$$

\footnotetext{
${ }^{14}$ Equilibrium does not pin down what the buyer must believe when it hears an unexpected report, but it does require that the beliefs must be concentrated on types that could feasibly have made that report.
} 
Proposition 1. In the persuasion game described above, suppose the three assumptions A1(a\&b) and A2 hold. Then, the following statements hold.

1. The buyer has a unique equilibrium buying strategy: $q^{*}(S)=\min \left\{q_{i} \mid S \in \Omega\left(\theta_{i}\right)\right\}$.

2. The buyer has a unique equilibrium belief - the skeptical belief-according to which $\pi_{S}^{*}\left(\theta_{i}\right)=1$ for $i=\min \left\{j \mid S \in \Omega\left(\theta_{j}\right)\right\}$.

3. The seller has many equilibrium reporting strategies $S^{*}$. A strategy is an equilibrium reporting strategy if and only if it has the property that $\min \left\{j \mid S^{*}\left(\theta_{i}\right) \in \Omega\left(\theta_{j}\right)\right\}=i$ for all $i=1, \ldots, S$..

4. In every equilibrium, the outcome is the same as the full information outcome: $q^{*}\left(S^{*}\left(\theta_{i}\right)\right)=q_{i}$.

The first statement of the proposition captures the main essence of the result. When the buyer hears a report the essence of which is that "the product quality might be $\theta_{i}$, which justifies a purchase of $q_{i}$, or it might be some state that justifies a higher purchase,” the buyer purchases only $q_{i}$ and never a greater quantity.

The second statement of the proposition indicates why that is the buyer's optimal response, namely, the report convinces her that the state really is $\theta_{i}$. If it were otherwise, then the seller would have said so. The buyer's extreme skepticism is a necessary property of equilibrium in this persuasion game.

The third statement asserts that when the state is actually $\theta_{i}$, the seller provides information indicating that the state index is at least that high and possibly higher. That 
distinguishes the seller from lower quality types. The ambiguity doesn't fool the buyer, but it doesn’t hurt, either.

Finally, the outcome of this persuasion game is a happy one: the buyer purchases just as she would if she were fully informed. If things were as simple as this, there would be no distortions and no use for regulations to govern the transaction and the release of information.

The argument used to prove Proposition 1 is commonly called the unraveling argument. The usual presentation is by mathematical induction, showing that the highest quality types always make reports that distinguish themselves and proceeding downward to lower quality types. The idea is that assumption A2 ensures that each quality type can distinguish itself and assumption A1(a\&b) assures that the highest quality types benefit by distinguishing themselves. That argument establishes statement 4 of the proposition and the rest then follow in short order.

To state the argument precisely, let us first argue that for any quality type $\theta_{i}$, the equilibrium sales must always be exactly $q_{i}$. Indeed, by assumption A2, the seller has a strategy that ensures the sales are at least $q_{i}$, so sales cannot be lower than that in any equilibrium. Also, in equilibrium, the buyer interprets information accurately, so if there is any quality type $\theta_{i}$ for which equilibrium sales are not $q_{i}$, then the equilibrium report $S=S^{*}\left(\theta_{i}\right)$ must also be made by at least one other quality type besides $\theta_{i}$. Let $\theta_{j}$ be the highest quality type that makes the report $S$; then, $S=S^{*}\left(\theta_{j}\right)$. By assumption A1(b), the sales when the report is $S$ must be strictly less than $q_{j}$, which implies that the seller is not maximizing sales when its quality type is $\theta_{j}$, contrary to the equilibrium condition. 
Hence, sales must coincide with the full-information buyer decision, proving statement 4 of the proposition.

Assumption A1(b) and the definition of equilibrium imply that $q^{*}(S) \geq$ $\min \left\{q_{i} \mid S \in \Omega\left(\theta_{i}\right)\right\}$. Suppose, contrary to statement 1 , that $q^{*}(S)>\min \left\{q_{i} \mid S \in \Omega\left(\theta_{i}\right)\right\}$ $=q_{j}$ for some $\theta_{j}$ with $S \in \Omega\left(\theta_{j}\right)$. Then, a seller of quality type is $\theta_{j}$ can sell more than $q_{j}$ by reporting $S$ and so must sell more in equilibrium. This contradicts statement 4 of the proposition, which we have already proved. Hence, statement 1 is also proved.

Statement 2 is the heart of the proposition. Since equilibrium beliefs must respect reporting feasibility, the buyer can assign positive probability only to quality types at least as high as $i=\min \left\{j \mid S \in \Omega\left(\theta_{j}\right)\right\}$. Suppose, contrary to statement 2 , that the buyer assigns strictly positive probability to any strict higher quality types. Then by assumption A1(b), the buyer will purchase a quantity $q\left(\pi_{S}^{*}\right)>q_{i}$. In that event, the seller with quality $\theta_{i}$ could sell more than $q_{i}$ by reporting $S$, contrary to the proven statement 4 .

Finally, statement 3 of the proposition characterizes the seller's possible equilibrium reports for each quality type as any reports that could not also be made by lower quality types. To see that is a necessary property of an equilibrium strategy, notice that if such mimicry by low quality types were possible, that would achieve sales for those types exceeding the full-information quantity, contradicting statement 4 . To see that the property is sufficient, we verify the equilibrium properties. The buyer must be optimizing, because she achieves her full information decisions for every quality type. Given the buyer's skeptical posture, no quality type of the seller can achieve higher sales. 
Finally, it is routine to check that the buyer's beliefs satisfy their two equilibrium conditions.

\section{How Uncertainty Mutes Skepticism}

When we introduce uncertainty about the seller's information, parts of the analysis change. The buyer still casts a skeptical eye on missing information, but her skepticism is muted because she is unsure about what information the seller could have reported. In terms of the buyer's purchase decision, this means that bad news is strictly worse than no news and leads to smaller quantity purchases. Understanding that, in equilibrium, the seller withholds bad news and reports only good news.

To capture these ideas in a simple model, we adapt a model of Shin (2003), assuming that the quality of the good is multidimensional and represented by $N$ characteristics. ${ }^{15}$ We denote by $x_{j}$ the good's score on characteristic $j$ and by $\chi_{i}=1$ and $\chi_{i}=0$ the events that the test of good $j$ is verifiable to the buyer. The entire vector $\left(x_{1}, \ldots, x_{N}, \chi_{1}, \ldots, \chi_{N}\right)$ is the seller's private information so a priori the buyer knows neither the realization of any test nor even whether there is a verifiable test result that could be reported.

In this model, a feasible report $S \in \Omega\left(x_{1}, \ldots, x_{n}\right)$ is a list of true statements reporting the product's score on some of the characteristics. To permit closed form solutions, let us suppose that the quality of the good is summarized by a scalar: $\theta=x_{1}+\ldots+x_{n}$ and that the buyer's value is $\theta v(q)$. Equilibrium is defined as above.

\footnotetext{
${ }^{15}$ The models introduced by Shin (1994) and Shin (2003) provide interesting treatments of how selective reporting of information of the sort considered here affect security price dynamics. Essentially, good information is readily reported while bad information dribbles out, which can (with additional assumptions) lead to predictions about asymmetric security returns and other interesting dynamics.
} 
For our analysis in this section, we replace assumptions A1(a\&b) by the stronger assumption A1. We replace A2, which restrict what the seller can verify, by the weaker assumption A3, which permits both that some characteristics may be unverifiable and that the buyer may be unable to identify those.

Assumption $A 3$. The $2 N$ random variables $x_{1}, \ldots, x_{N}, \chi_{1}, \ldots, \chi_{N}$ are statistically independent. For each $j, x_{j}$ is uniformly distributed on $[0,1]$ and the probability that $x_{j}$ is verifiable is $\operatorname{Pr}\left\{\chi_{j}=1\right\}=\pi_{v}$. The seller observes the realizations of these variables but the buyer does not.

Proposition 2. Assume A1 and A3. Then there is a unique equilibrium $\left(S^{*}, q^{*}, \pi^{*}\right)$ of the persuasion game with the property that the buyer's assessment of each $x_{j}$ depends only on the report about $x_{j}$. Let $\bar{x}=\left(\sqrt{1-\pi_{v}}-\left(1-\pi_{v}\right)\right) / \pi_{v}$. Then, in this equilibrium,

1. According to $S^{*}$, the seller reports the outcome of a test $j$ if and only if $\chi_{j}=1$ and $x_{j}>\bar{x}_{j}$.

2. Given any report $S$, the buyer's posterior belief is $\pi_{S}^{*}$, according to which the various test results are statistically independent and have the following probabilities

\footnotetext{
a. If the seller reports that $x_{j}=\alpha$, then $\pi_{s}^{*}\left(\left\{x_{j}=\alpha\right\}\right)=1$.

b. If the seller makes no report about test $j$, then for any $\alpha \in[0,1]$,

$$
\pi_{S}^{*}\left(\left\{x_{j} \leq \alpha\right\}\right)=\left\{\begin{array}{cc}
\alpha /\left(\bar{x}+(1-\bar{x})\left(1-\pi_{v}\right)\right) & \text { for } \alpha \leq \bar{x} \\
\left(\bar{x}+(\alpha-\bar{x})\left(1-\pi_{v}\right)\right) /\left(\bar{x}+(1-\bar{x})\left(1-\pi_{v}\right)\right) & \text { for } \alpha>\bar{x}
\end{array} .\right.
$$
}


3. The buyer purchases the quantity $q^{*}(S)=q\left(\pi_{S}\right)$.

The key to interpreting of Proposition 2 lies in the buyer's beliefs when no verifiable test result is reported for test $j$. The formula is simply a conditional probability calculation. In the numerator is the joint probability that the true test value is less than $\alpha$ and no report is made. In the denominator is the probability of the event that no report is made. For low values of $\alpha$, the joint probability is the same as the probability that the test value is less than $\alpha$. For high values of $\alpha$, it is the probability that the test value is too low to lead to a report plus the probability that the test value is high enough, but still less than $\alpha$ and that no verifiable report is possible.

Unlike our first model, this one leaves a potential role for liability rules or direct regulation, because firms do sometimes withhold information. For suppose a court or regulator could determine after the fact that a firm withheld negative information and could impose liability for any losses. Then, more complete information would be revealed to the buyer, improving buyer decision making.

The model of this section, however, is still too thin to enable actual welfare analyses. It omits the seller's decision about whether to test at all, which our motivating examples suggest is an important part of the story. To focus attention on that, we specialize the model and add more detail about the buyer's and seller's payoff. This enables a meaningful welfare analysis and also allows us to begin our examination of the seller's incentive to perform verifiable tests which might mitigate the problem of adverse selection. 


\section{The Seller's Incentive to Disclose}

Consider next an extended persuasion game in which, in addition to making a report $S$, the seller also sets the price $p$ of its product. Suppose that the buyer is making a yes-or-no decision to buy a fixed number of units of the product; for example, the number of new furnaces she will buy is either zero or one. Using the notation of the preceding section, $v(\theta)$ is the consumer's full-information reservation price-the highest price she would pay for a unit of the good. In equilibrium, the seller can work out how its report $S$ will affect the reservation price, so it sets the product price to $p=E[v(\theta) \mid S]$ and extracts the whole surplus as its profit.

Suppose now that reporting is costly, that is, the seller must incur a cost to make a verifiable test of attribute $j$. Intuitively, the seller's incentive to test and reveal information is at least sometimes excessive. An extreme example illustrates the possibility and highlights some generalizable economics. Thus, suppose there is just one relevant product attribute ( $N=1$ ) and that the product's marginal cost is $\gamma=0$. Then, the buyer is willing to purchase at a price above cost even if no information is reported. A planner who can regulate the amount of verifiable testing but cannot regulate the product price would recognize that the value of information in this situation is zero, so it could achieve the total-surplus-maximizing outcome by proscribing all costly verifiable reporting. With such a regulation, the seller would set the price to $p=E[v(\theta)]$, which makes the buyer just willing to purchase the product, and total surplus would be maximized. In particular, no resources would be wasted on verifiable testing that affects the price but not the allocation of the product. 
Despite the fact that costly testing can only reduce the total surplus in this example and that, at equilibrium, the consumer's payoff cannot be negative, the seller cannot avoid doing some testing in equilibrium if the testing cost is sufficiently low. For suppose that the no testing outcome always occurs. Since the buyer would then expect no testing, the product price $p$ would be set to $E[v(\theta)]$ and the seller would enjoy that entire amount as its producer surplus. When quality is sufficiently good $\left(\theta=\theta_{N}\right)$ and testing is sufficiently cheap $\left(c<v\left(\theta_{N}\right)-E[v(\theta)]\right)$, the seller could then increase its net profit to $v\left(\theta_{N}\right)-c>E[v(\theta)]$ by testing, reporting, and raising its price to $v\left(\theta_{N}\right)$. That profitable deviation contradicts the possibility of an equilibrium with no testing.

There are several suggestive points in this example, but let us state them for a situation in which an uninformed buyer would not want to buy at all. Throughout, we assume that testing is costly, so the planner's optimum would involve less than complete revelation. In such a situation, the planner would want to reveal product information only when (1) trade actually adds positive value and (2) the amount of value added, which is $v(\theta)-\gamma$, exceeds the cost of testing. The planner would want the seller to use only the least costly verifiable test sufficient to cause the purchase to proceed.

The seller's incentives are partially aligned with that, but with two important differences. First, since the price-setting seller captures the whole surplus from any transaction (the buyer just breaks even), it prefers testing over no-testing whenever the planner does. The seller may also want to test, however, when the product is actually so bad that there are no net gains from trade but where there is enough favorable 
information to convince the buyer anyway. So, the seller tests any time the planner would test, and other times, too.

Second, for every level of quality, the seller will always want to spend at least as much on testing as the planner, and sometimes more. This is obvious for the case where the planner would prefer to do no testing. When the planner prefers some testing, it would like to do that in the least cost way which causes the transaction to proceed. The seller's interests are different. Besides wanting the transaction to proceed, it wants also to get the highest possible price net of its costs and that generally calls for revealing different information than the planner would prefer. In many of our simple models, “different” translates into "more” because additional good news can only raise the price of the product.

Both of these observations amount to saying that verifiable testing combined with selective reporting creates a pervasive negative externality in equilibrium. We have already described this externality in the introduction. Holding the buyer's beliefs fixed, suppose the seller runs an additional test and reports its results selectively and compare that to the situation where no additional test is conducted. If the test result is good, that allows the seller to charge a higher price; if it is bad, the seller withholds the report and gets the same price as if no test had been run. Hence, with selective reporting, extra testing leads to higher average prices. Selective reporting thus leads to a negative pecuniary externality which encourages excessive testing.

In equilibrium, buyers form their beliefs correctly, so the seller's manipulations do not lead to systematically high prices and losses for the buyer. Instead, the equilibrium is a sort of rat race similar to that found by Akerlof (1976), in which the seller's effort to 
extract rents raises costs unnecessarily and causes a loss of value that the seller itself bears.

Our findings are summarized in the first three points of the following proposition.

Proposition 3. In equilibrium of the persuasion-and-pricing game,

1. Prices are set so that the buyer's expect profit, conditional on its information, is always zero: $p_{S}=E[v(\theta) \mid S]$.

2. For each level of quality, the seller spends as much or more on testing and verification as the amount that would be spent by a total-surplusmaximizing planner.

3. Overspending on testing occurs whenever the product quality is sufficiently high and the cost of additional testing is sufficiently low.

4. If the cost of reporting each characteristic is $c \in[0,1 / 2]$ and $v(\theta)=\theta=\sum_{j=1}^{N} x_{j}$, then the seller tests and reports characteristic $j$ whenever $x_{j} \geq 2 c$ and, given any set $R$ of reported characteristics, the price charged is $\sum_{j \in R} X_{j}+(N-|R|) C$.

Only the fourth point of the proposition has not yet been explained and it merely explains the seller's and buyer's strategies in a particularly simple special case. It is intuitive that, in equilibrium, the seller reports characteristic $j$ whenever $x_{j}$ is sufficiently large, say $x_{j} \geq \bar{x}$. The buyer who sees no report about $x_{j}$ makes a correct inference and infers, based on the uniform distribution, that the expected value of the unreported 
characteristic is $1 / 2 \bar{x}$, so the buyer will pay at most $\sum_{j \in R} x_{j}+1 / 2(N-|R|) \bar{x}$ and the seller sets the price accordingly. Given the buyer's strategy, the seller prefers to incur the cost $c$, report the true $x_{j}$, and be paid accordingly when $x_{j}-c \geq 1 / 2 \bar{x}$. The value of $\bar{x}$ that renders the two inequalities identical is $\bar{X}=2 c$.

The models underlying Propositions 2 and 3 assume that the seller is fully informed about its product. In that situation, if the product characteristics could be costlessly verified after consumption, then costly pre-sale verification would be unnecessary, because a liability rule that holds the seller responsible after-the-fact for any false statements would deter dissembling. That sort of ex post verification effectively returns us to the situation modeled in Proposition 1, in which buyer skepticism leads to full disclosure and there is no need for regulation.

There are two reasons why, in practice, ex post verification of claims is unlikely to provide sufficient protection for buyers. One is asymmetric information. For example, a pharmaceutical company may be aware that patients using its new drug are experiencing poorer results than had been expected, but it may not yet have run tests or evaluated the clinical data to determine the nature or extent of the problem. Similarly, a firm issuing new shares may be aware of complaints about its employment or environmental policies, but it may not yet have evaluated what legal risks those policies create. In these situations, the seller arguably has no verifiable information that is governed by simple mandatory disclosure rules and it may fear that generating such information can only lead to negative results. Some valuable testing and disclosure would then be suppressed. 
A second reason why ex post verification of claims may be insufficient to restore efficiency is that the buyer may not know what to ask or how to interpret the information she has. A seller of ceiling insulation may fail to report that the product contains asbestos or another dangerous chemical and the buyer, even if she has a sophisticated understanding of the strategic situation, may simply not have the product knowledge or scientific knowledge to know what information to expect, what questions to ask, or what to make of a report that a product does contain a certain chemical.

\section{When Revelation Should be Compelled}

Regulatory remedies for situations like those described above may need to be intrusive in markets or more difficult to administer than merely mandating reporting of known test results. For instance, in the pharmaceutical industry example, the seller uses its unverifiable private information to make decisions about which tests to perform. Regulation that tries to force testing ex ante falters on an information problem: if the regulator knew which of the missing tests to compel, then the buyer could equally well simply be skeptical about the absence of those tests. The alternative to compelling testing is to impose liability rules, but these must be different from the ones discussed above, because the problem is not one of false reporting. What is needed to restore incentives is to hold the seller liable for failures to reveal promptly not only the verifiable information that the seller knew but also the information that it should have known. This works better than compelling immediate disclosure if, eventually, accumulating evidence about a product defect comes to light and if discovery in a legal proceeding can then reveal when the seller became aware of a possible problem. In practice, such a system can work only 
it is eventually possible to establish what the seller should have known and when it should have known it.

The second example, in which consumers don't know the relevant product science, has different potential remedies. The simplest solution, in principle, is for an industry regulator who is an expert in the subject matter to mandate the relevant material disclosures. ${ }^{16}$ Such regulations are sometimes attempted. For example, there have long been regulations requiring life insurance companies that sell whole life insurance policies to forecast the policy’s cash value accumulations and net realized costs at various future dates, although these forecasts rely on many assumptions which are not guaranteed. As shown below, competition among sellers is sometimes a partial substitute for regulation in this kind of setting, because it can compel sellers to reveal sufficient information to enable buyers to make good decisions.

Game theory, with its standard assumptions of rationality, does not yet have a really good way to accommodate models of buyers who are so unaware of relevant facts that they cannot even reason about them. The buyer who has never even heard of asbestos is different from a buyer who does not know that it is risky, but she cannot even ask a question about it. Nevertheless, some progress in modeling unaware consumers can be made by the following sort of model.

Suppose that there are $N \geq 2$ product characteristics, but only one characteristic actually varies in quality. Instead of unawareness, we impose limits on communication of

\footnotetext{
${ }^{16}$ Even here, ex post regulation has a role. Laws against fraud, implied warranties of merchantability or product fitness all aim to mitigate reporting and adverse selection problems.
} 
technical information, so the seller can report test results about only a small number of characteristics, say just one.

Although verifiable tests are available for every characteristic, the buyer's utility from purchasing the product depends on only one of the characteristics. If the relevant characteristic is good (which occurs with probability $\pi$ ) or if the characteristic is not the relevant one, then its test result is 1 . We assume that the seller can report any test value less than or equal to the actual test value. ${ }^{17}$ If the relevant characteristic is bad (which has probability $1-\pi$ ), then its test result is uniformly distributed on the interval $[0,1]$. The seller can verifiably report any value less than or equal to the test result. The game proceeds by the seller naming one characteristic and verifiably reporting its test result.

Finally, we model the idea that the buyer is a "novice" by assuming that she does not know the names of any characteristics until the seller reports one, so she cannot determine whether the characteristic the seller reports about is the relevant characteristic or some other one. ${ }^{18}$

Proposition 4. In the persuasion game with a novice buyer, there is an equilibrium in which any report is about a test result of value 1 , the buyer regards any report as uninformative and chooses $q$ accordingly.

If the buyer is informed enough to name some characteristics, then there is a better procedure which, in equilibrium, conveys some useful information. According to this procedure, the buyer asks the seller for information about some characteristic that

\footnotetext{
${ }^{17}$ This "free disposal in reporting" is included so that a seller cannot establish the relevance of its test report merely by reporting a number less than 1 -say 0.99 , to establish that the relevant characteristic is actually quite good.

${ }^{18}$ This novice buyer model is adapted from Milgrom and Roberts (1986).
} 
she, the buyer, can name. With probability $1 / N$, that is the relevant characteristic and, if the product is actually bad, the seller's verifiable report (or the seller's refusal to make any report) will establish that. If the seller responds with a good report, the buyer's decision can also account probabilistically for the information that such a report conveys.

The persuasion game with a novice buyer (or an “unaware” buyer) creates the possibility that an expert regulator could, by requiring product labeling for relevant characteristics, encourage more useful and informative disclosures. Labeling emerge as remedies in our analysis when consumers are novices, that is, so unfamiliar with the product that they don't know what to ask about, and when all consumers have the same concerns. With too much consumer heterogeneity, the labeling solution fails, because the regulator does not know which characteristic needs to be reported. Mandating full reporting fails because the buyer has limited information processing capacity.

Another solution that could, in principle, restore efficient arises when there are some agreed product standards, the seller is required to disclose product defects, and there is ex post liability for failures to disclose. Consumer heterogeneity, however, is again a problem, particularly if the buyer's characteristics can change over time. In such cases, it may be difficult to verify whether the seller had reported the right information for the particular buyer. Reporting too much information in this situation leads to information overload, in which the buyer may fail to notice the most relevant information.

\section{Regulation or Competition?}

Our example of the furnace purchase is typical of ones in which buyers may differ in their relevant characteristics, because the optimal furnace depends on other 
characteristics of the buyer's home. The size of the home, the capacity of hot air ducts, the amount of insulation, the construction of the chimney, and other factors can all be things about which a buyer might need to be informed. If a regulator can select just one, which one should it be? Surely, it is better to have an expert seller be motivated to report the relevant characteristic. When can competition among sellers create such an incentive?

Not always. For example, it may be that no furnace seller will report to the buyer that her best choice is to repair the old furnace or install better insulation, or for a tobacco company to report that all tobacco products can cause cancer and lung disease. However, if we set the more limited objective of having the buyer make the best choice from among the sellers' offerings and if each seller offers only a single product (or if the product price is endogenous), then sellers with the best offerings will be motivated to point out the characteristics that most distinguish their own products. Competition can lead to useful disclosures, if not complete ones, even to novice buyers.

To study this sort of competition in a formal model, we modify the novice buyer model of the preceding section. Suppose there are two sellers, both of which are perfectly informed about the relevant product characteristics and about how each product scores on that characteristic. Each seller names a characteristic, which we interpret as the claim that it is the relevant one. The buyer then identifies a characteristic that one of the sellers has named. We interpret this as a request that both sellers report about that characteristic. Each seller may then make a verifiable report about the identified characteristic or about the one they have named. Finally, the sellers quote prices for their products and the buyer chooses one or neither. For simplicity, the sellers' supply cost for the product is zero. 
Because the buyer's announcement is pure cheap talk, this game necessarily has multiple equilibria. One that is most consistent with our interpretation of the moves is as follows.

Proposition 5. The following is an equilibrium of the persuasion game with competing sellers and a novice buyer.

1. If a seller has a better test report for the relevant characteristic than the other seller, then it names the relevant characteristic to the buyer at the first round of the game. Otherwise, the seller names any characteristic on which it gets a perfect test report (test value $=1$ ).

2. The buyer randomizes and selects one of the two named characteristics, each with equal probability.

3. The sellers each report their test result on the buyer's named characteristic. Each seller also names its corresponding Bertrand equilibrium price for the subgame, given the buyer's information.

4. The buyer beliefs are formed as follows. If a seller reports the test result for the buyer-identified characteristic, then the buyer believes that report is equal to the test result. If a seller fails to name the required characteristic, then the buyer infers that its test result for the buyer-identified characteristic is zero.

5. Given its beliefs, the buyer determines its values for the two products. It buys the product for which value minus price is greatest. In the event of a tie, if one product has a higher quality than the other, then the buyer 
purchases the higher quality product, and otherwise it selects one of the two products at random.

In this equilibrium, the buyer gets some information from competition among the sellers. The seller with the better quality product names the relevant characteristic in order to win the sale, even if that depresses its price somewhat. Compared to the regulatory solution, if the relevant characteristic can vary among buyers, competition can lead to more relevant and valuable reporting. In a variation of the model where the buyer can ask about both characteristics, the full-information efficient outcome results. Generally, however, so long as the buyer capacity to process information is limited relative to the amount of information reported, some inefficiency of decisions is to be expected.

\section{Conclusion}

When buyers are sophisticated, markets provide powerful incentives for sellers to provide verifiable product information. This can sometimes go a long way toward alleviating problems of adverse selection. Yet, sellers may still have an incentive to test selectively, based on their unverifiable suspicions about which tests will make their products look good. Regulation, especially in the form creating liability for withholding material information, can help to mitigate the costs of non-disclosure.

The problem becomes harder when buyers are novices, that is, when they are unsure about which product characteristics are relevant to them and when complete information about the product is just too complex and detailed for the buyer to analyze. If buyers are not too heterogeneous, this can sometimes be solved by expert regulators who mandate disclosure of the most significant product characteristics, but that can become 
too difficult when buyer heterogeneity causes the number of potentially significant characteristics to multiply. Competition among sellers helps somewhat, because sellers will be led to emphasize the important characteristics on which their own products have an advantage. If, however, the best decision for a buyer lies outside the set offered by the sellers, then no seller will have an incentive to reveal this fact. 


\section{References}

Akerlof, George. 1976. "The Economics of Caste and of the Rat Race and Other Woeful Tales." Quarterly Journal of Economics, 90:4, pp. 599-617.

Crawford, Vincent P. and Joel Sobel. 1982. "Strategic Information Transmission." Econometrica, 50:6, pp. 1431-51.

Dye, Ronald. 1985. "Disclosure of Nonproprietary Information." Journal of Accounting Research, 23:1, pp. 123-45.

Farrell, Joseph and Matthew Rabin. 1996. "Cheap Talk." Journal of Economic Perspectives, 10:3, pp. 103-18.

Frankel, Richard, Maureen McNichols, and G Peter Wilson. 1995. "Discretionary Disclosure and External Financing." Accounting Review, 70:1, pp. 135-50. Glazer, Jacob and Ariel Rubinstein. 2006. "A Study in the Pragmatics of Persuasion: A Game Theoretical Approach." Theoretical Economics, 1, pp. 395-410.

Grossman, Sanford. 1981. "The Informational Role of Warranties and Private Disclosure About Product Quality." Journal of Law and Economics, pp. 461-83.

Grossman, Sanford and Oliver Hart. 1980. "Disclosure Laws and Takeover Bids."

Journal of Finance, 35:2, pp. 323-34.

Jovanovic, Boyan. 1982. "Truthful Disclosure of Information." Bell Journal of

Economics, 13:1, pp. 36-44.

La Porta, Rafael, Florencio Lopez-de-Silanes, and Andrei Shleifer. 2006. "What Works in Securities Laws?" Journal of Finance, 41:1, pp. 1-32.

Milgrom, Paul. 1981. "Good News and Bad News: Representation Theorems and Applications." Bell Journal of Economics, 12, pp. 380-91.

Milgrom, Paul and John Roberts. 1986. "Relying on the Information of Interested Parties." Rand Journal of Economics, 17, pp. 18-32.

Shin, Hyun Song. 1994. "News Management and the Value of Firms." Rand Journal of Economics, 25:1, pp. 58-71.

Shin, Hyun Song. 2003. "Disclosures and Asset Returns." Econometrica, 71:1, pp. 10533.

Topkis, Donald. 1978. "Minimizing a Submodular Function on a Lattice." Operations Research, 26:2, pp. 305-21.

Verrecchia, Robert. 1983. "Discretionary Disclosure." Journal of Accounting and Economics, 5, pp. 179-94. 